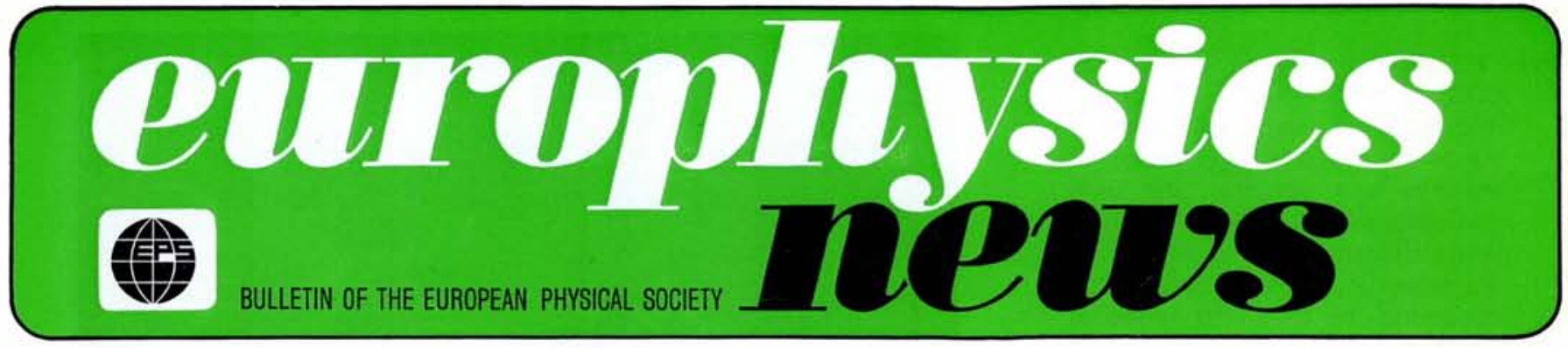

\section{Introduction}

The "Convention for the Establishment of a European Space Research Organisation" was signed in 1962 by the ten Member States (Belgium, Denmark, France, Fed. Rep. Germany, Italy, The Netherlands, Spain, Sweden, Switzerland and the United Kingdom), and entered into force on 20 March 1964. Under its terms, the purpose of the Organisation is to provide for, and to promote, collaboration among European states in space research and technology, exclusively for peaceful purposes. ESRO has had an important role in stimulating technological development in industry in its Member States.

Until 1971, the Organisation's activities were devoted mainly to the development and launching of satellite and sounding-rocket payloads for scientific purposes, with a supporting programme of research and development. During 1971, however, a detailed review of the Organisation's modus operandi was carried out to prepare for extension of its activities to the applications field and to provide greater programme flexibility. The decision to enter the applications field meant that, in future, less money would be available for scientific activities; this led to the decision to terminate the Organisation's sounding-rocket activities (although certain Member States continue these at Kiruna (Sweden) by means of a special project arrangement with the Swedish Government) and to cease the fundamental research work in space physics that had been carried out at the European Space Research Institute (ESRIN) near Rome.

The scientific programme and certain basic activities are financed by all Member States in proportion to their Gross National Products, whereas participation in all other programmes is optional. The main contributors to the obligatory programme are Fed. Rep. Germany $(23.16 \%$ in 1973$)$, France $(21.39 \%)$ and the United Kingdom $(18.60 \%)$.

The Organisation at present has a staff of about 1200 , headed by a

\title{
The European Space Research Organisation
}

\section{R. J. Davidson, ESRO}

Director General (A. Hocker). Implementation of the Organisation's policy, as laid down by the Council and its committees, is in the hands of a Directorate of five, comprising the Director General and the Directors of Programmes and Planning, Administration, the European Space Research and Technology Centre (ESTEC) and the European Space Operations Centre (ESOC). The first three of these Directors are located at the Organisation's Head Office in Neuillysur-Seine, near Paris. ESTEC, which is responsible for all study and development work on satellites, is at Noordwijk, The Netherlands, whilst ESOC, which is responsible for satellite orbit and attitude determination, tracking, data acquisition and processing, is at Darmstadt, Fed. Rep. Germany.

\section{Current development programme}

\section{(a) Scientific Satellites}

At the beginning of 1973, the scientific satellite programme consisted of the COS-B, GEOS and IUE (International Ultraviolet Explorer) projects, the latter being a joint NASA/UK/ESRO venture. In April 1973, however, the ESRO Council approved two additional projects, namely HELOS (High-Eccentricity Lunar Occultation Satellite) and participation with NASA in the IME (International Magnetospheric Explorer) programme. Thus the following five scientific satellite projects are now under way :

(i) COS- $B$ will carry a single, complex experiment for studying the origin and energy spectrum of extraterrestrial gamma-radiation. The various units constituting the experiment are being provided by six European scientific laboratories, including ESTEC's Space Science Department. The development contract was placed early in 1972 and the scheduled launch date is February 1975.

(ii) GEOS, as its name implies, is a geostationary satellite, and will carry seven experiments for the study of particle fluxes, electric and magnetic fields and electromagnetic waves in the far magnetosphere. The development contract was placed in May 1973, with launch scheduled for the summer of 1976 .

(iii) IUE, (formerly known as SAS-D), is planned for launch into a geosynchronous orbit in December 1976. It is, in essence, an ultraviolet astronomy observatory being developed by NASA for international use. ESRO will contribute the deployable solarcell array and will design, build and operate a European ground station, in return for which European astronomers will be allocated a percentage of the available observation time.

\section{Contents}

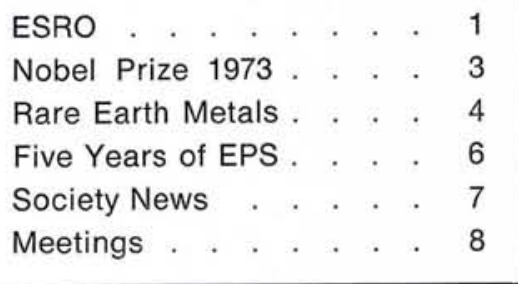


(iv) EXOSAT (formely known as HELOS), scheduled for launch in mid-1979, will carry out measurements of the position, structural features and spectral and time characteristics of X-ray sources. It will determine the position and diameter of celestial objects by observing the time and speed at which they disappear behind the Moon.

(v) IME is a three-satellite project. ESRO is developing one of the two satellites which will be launched by the same rocket in 1977 and will follow each other along a roughly equatorial orbit with an apogee of about 23 Earth radii. With an inter-satellite separation of 100 to $5000 \mathrm{~km}$, these satellites will be able to differentiate between temporal and spatial phenomena in the magnetosphere. The third, a Heliocentric satellite, will remain at about 250 Earth radii from the Earth and will accompany it around the Sun. It will thus be able to monitor the solar wind undisturbed by the presence of the Earth, and will provide "baseline" inputs to the analysis of data from the other two. All three satellites will carry both US and European experiments.

\section{(b) Applications Satellites}

In December 1971, three ESRO applications programmes were approved: Meteosat, Aerosat and the European Communications Satellite (ECS). Studies are also being made on "second-generation" applications missions such as Earth-resource surveys and TV and radio broadcasting.

(i) Meteosat programme. As a contribution to the World Weather Watch organised by the World Meteorological Organisation, ESRO intends to launch a meteorological satellite around the end of 1976. In geostationary orbit, stationed between $0^{\circ}$ and $30^{\circ} \mathrm{E}$, the satellite will carry an Earth-observation package and a data transmission and relay package. The main objectives are simultaneous half-hourly imaging of the Earth's surface and cloud system in the visible and infrared, transmission of raw image data to major user stations, relay of narrow bandwidth weather (WEFAX) data to small user stations, and data interrogation and collection from up to 4000 drifting and fixed Earth-based sensor platforms.

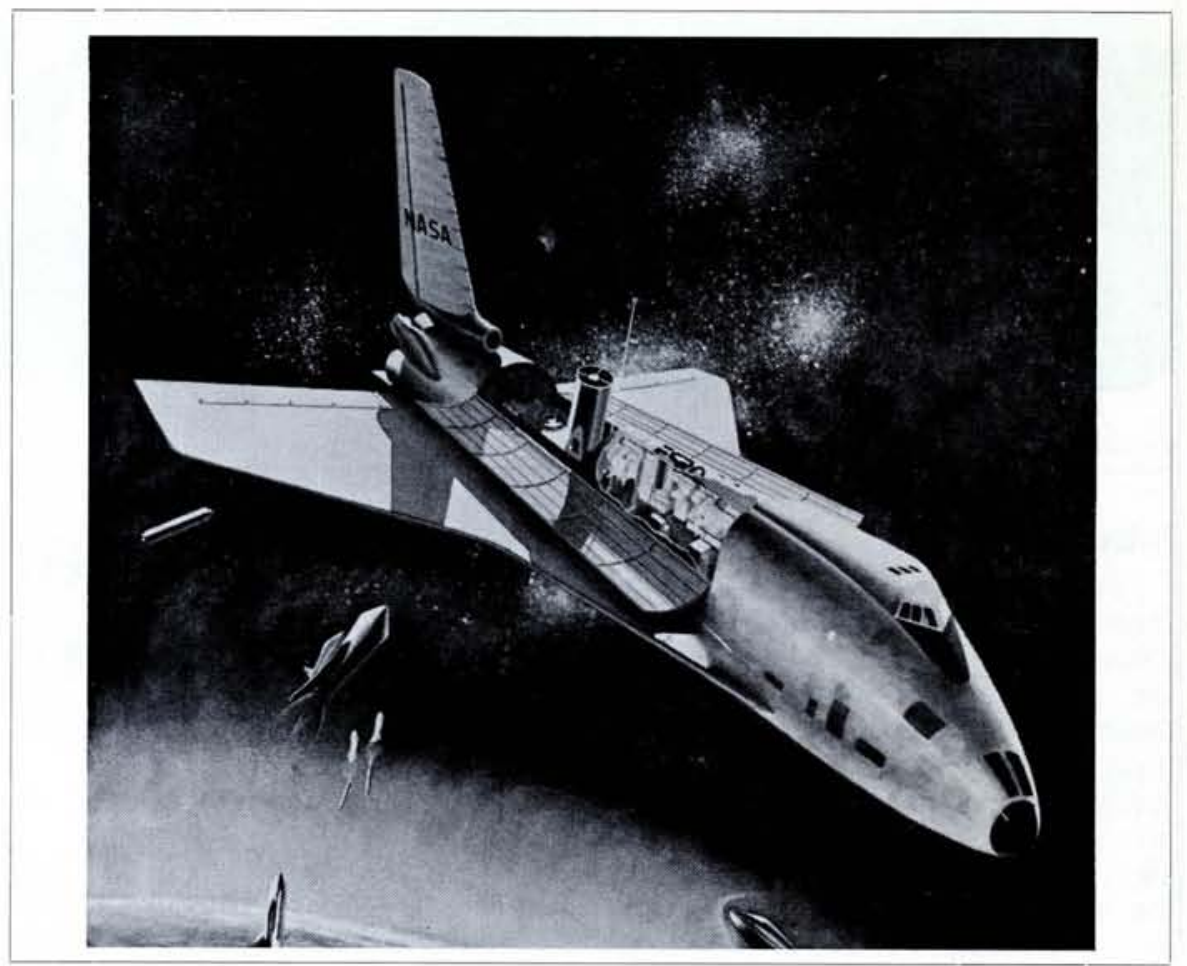

Spacelab, the ESRO contribution of the post-Apollo programme undertaken by NASA. 50 missions of scientists and engineers (in average-sized crews of four) will use Spacelab during its 10-year operational lifetime.

(ii) Aerosat programme. This preoperational aeronautical satellite system's main objective is to evaluate the advantages of space techniques in resolving the problems of air traffic control over oceans by supplying communication and localisation data to ground control centres. Negotiations on an agreement between the US, Canada and Europe have been in progress for some time, while the possibility of undertaking the programme on a different basis was kept open by means of work executed for ESRO by European industry.

(iii) ECS programme. The overall aim of ESRO's programme of telephony and television distribution satellites is to provide the Conférence européenne des Postes et Télécommunications (CEPT) and the European Broadcasting Union, by the end of the present decade, with a system enabling them to route part of their circuit requirements via space. The programme is now well into its second (technological and development) phase, covering the development of an Orbital Test Satellite (OTS) for testing critical units and techniques, ground tests of the communication system and a large-scale supporting technology programme. Competitive design and definition studies of OTS are at present in progress and the satellite development contract is due to be awarded in December 1973, with launch scheduled three years later. As part of the overall experimental phase, ESRO is also providing certain items of equipment for the Canadian Communications Technology satellite, due to be launched in 1975.

\section{The future}

In addition to the five scientific and three applications programmes outlined above, together with a strong supporting technology programme, the recent decision by Ministers at the European Space Conference on 31 July 1973 to proceed with three other major projects has emphasised Europe's strong desire to become a serious force in international space technology.

Ministers approved three major new inter-dependent projects - Space -lab, the LIIIS launcher and the MAROTS satellite - and decided to set up a single European Space Agency (ESA) by 1 April 1974.

Considerable progress has already been made in the studies on Spacelab, which represents Europe's contribution to the US post-Apollo programme. Competitive detailed definition studies now being undertaken by European industry under the leadership of the firms of MBB and 
ERNO will be completed early in 1974. Award of the development contract is scheduled for mid-1974. Spacelab will comprise a pressurised laboratory providing a shirt-sleeve environment, and an unpressurised pallet for mounting experiments requiring direct exposure. The complete unit, weighing a maximum of about $14000 \mathrm{~kg}$, will be transported to and from Earth orbit in the payload bay of NASA's shuttle orbiter, and will remain attached to, and supported by, the orbiter throughout each mission. It will be designed for an operational lifetime of 10 years (50 missions, each of seven day's duration, with ground refurbishment). Its major advantages are its reusability and the fact that scientists and engineers (in average-sized crews of four) will be able to go along on the flight to control the experiments and subsystems, and bring their data back with them.

The second of the newly approved projects, the LIIIS launcher, is in- tended to provide Europe, by 1980 , with an independent capability for putting geostationary satellites into orbit. The three-stage launcher will be capable of placing in transfer orbit payloads of $1500 \mathrm{~kg}$, enabling the injection of satellites of some $750 \mathrm{~kg}$ into geostationary orbits with the aid of a suitable apogee motor. The project was put forward by France, following termination of the Europa-ill programme, and the French national space agency, CNES, has already started work on certain parts of the definition phase which will be completed by the end of 1973 . Until the establishment of the European Space Agency, ESRO will liaise between its Member States participating in the LIII S programme and CNES.

The third project, MAROTS (Maritime Orbital Test Satellite), has been generated by the considerable growth of interest in maritime satellites in the last two years. In the U.K., a Geostationary Technology Satellite
(GTS) programme, originally intended for telecommunication purposes, was re-oriented to meet requirements for aiding maritime navigation, and in April 1973 the U.K. proposed that the programme should be Europeanised, taking into account the stage reached at national level. In the meantime, ESRO had been studying the possibility of adapting the telecommunications OTS vehicle for use in a maritime role (the MAROTS proposal). At the European Space Conference meeting on 31 July 1973, the Ministers agreed on the MAROTS proposal, and details of the programme management, etc., are now being worked out.

It is worth noting that each of the three major contributors has undertaken to provide more than half of the financial backing for one of the three new programmes (Germany $52.55 \%$ for Spacelab, France $62.50 \%$ for the LIII S, and the U.K. $56 \%$ for MAROTS).

\section{Nobel Prize in Physics 1973}

\section{H. R. Zeller, BBC, Baden}

This year's Nobel prize for physics was awarded to Leo Esaki (IBM, USA), Ivar Giaever (General Electric, USA) and Brian Josephson (Cambridge, England) for their pioneering work in the field of electron tunneling.

In the early days of quantum mechanics, it was shown that a particle can pass across a potential barrier even if classically its energy is insufficient. This effect became known as the tunnel-effect and the related theory was developed by Gamov in connection with $\alpha$-decay where the $\alpha$-particles tunnel across the Coulomb barrier of the nucleus. In the late fifties, Esaki and Giaever introduced tunneling into solid state physics.

The tunnel diode invented by Esaki consists of a p-n junction in which both $\mathrm{n}$ and $\mathrm{p}$ side are heavily doped to make the material degenerate and the transition sharp. For small voltages the electrons in the conduction band of the n-material directly tunnel into the empty states of the valence band of the p-side. At higher voltages no energy conserving tunneling pro- cesses are possible due to the lack of empty states on the opposite side of the junction. This results in a negative resistance region. It is this negative resistance region on which the technical applications of the Esaki diode are based. By slightly oxidizing a metal strip and subsequent vapour deposition of a cross strip, Giaever produced a sandwich consisting of two metal films separated by a thin (20 - 50 A) oxide barrier. Tunneling takes place through the oxide barrier and results in a small-voltage conductivity of the order of $1 \Omega \mathrm{mm}^{-2}$ of the oxide.

The first scientific success of the new technique was Giaever's demonstration of the validity of the BardeenCooper-Schrieffer (BCS) theory of superconductivity. Both the existence of an energy gap and the particular density of states predicted by the BCS theory were experimentally verified.

The tremendous success of tunneling in superconductivity suggested that it could be used more generally as a probe to study all sorts of excitations of the solid state. Subsequent work showed in fact that it was possible to observe molecular vibrations, phonons, plasmons, magnetic excitations, etc., in a very simple form of spectroscopy by voltmeter. But it also became evident that, with the exception of superconductivity, tunneling is mainly probing surface and not bulk properties.

Although the Josephson effect is not necessarily related to tunneling, it was first experimentally observed in tunnel junctions. Josephson showed that if two superconductors are weakly coupled, for instance by the tunnel effect in a superconductorinsulator-superconductor sandwich, there will be a phase relation between the two superconductors. In other words, the whole structure may behave as one superconductor with a supercurrent flowing through the oxide. Generally speaking, the Josephson effect is an example of a macros- 